\title{
A New Optimal Eighth-Order Ostrowski-Type Family of Iterative Methods for Solving Nonlinear Equations
}

\author{
Taher Lotfi ${ }^{1}$ and Tahereh Eftekhari ${ }^{2}$ \\ ${ }^{1}$ Department of Applied Mathematics, Hamedan Branch, Islamic Azad University, Hamedan 65138, Iran \\ ${ }^{2}$ Faculty of Mathematics, University of Sistan and Baluchestan, Zahedan 987-98155, Iran
}

Correspondence should be addressed to Taher Lotfi; lotfitaher@yahoo.com

Received 6 September 2013; Accepted 2 October 2013; Published 13 March 2014

Academic Editors: Q. Guo and Z.-Y. Li

Copyright ( 2014 T. Lotfi and T. Eftekhari. This is an open access article distributed under the Creative Commons Attribution License, which permits unrestricted use, distribution, and reproduction in any medium, provided the original work is properly cited.

Based on Ostrowski's method, a new family of eighth-order iterative methods for solving nonlinear equations by using weight function methods is presented. Per iteration the new methods require three evaluations of the function and one evaluation of its first derivative. Therefore, this family of methods has the efficiency index which equals 1.682. Kung and Traub conjectured that a multipoint iteration without memory based on $n$ evaluations could achieve optimal convergence order $2^{n-1}$. Thus, we provide a new class which agrees with the conjecture of Kung-Traub for $n=4$. Numerical comparisons are made to show the performance of the presented methods.

\section{Introduction}

In this paper, we consider iterative methods to find a simple root $\alpha$ of a nonlinear equation $f(x)=0$, where $f: D \subset \mathbb{R} \rightarrow$ $\mathbb{R}$ is a scalar function on an open interval $D$. This problem is a prototype for many nonlinear numerical problems. Newton's method is the most widely used algorithm for dealing with such problems, and it is defined by

$$
x_{n+1}=x_{n}-\frac{f\left(x_{n}\right)}{f^{\prime}\left(x_{n}\right)}
$$

which converges quadratically in some neighborhood of $\alpha$ (see $[1,2])$.

To improve the local order of convergence, many modified methods have been proposed in the open literature; see [3-17] and references therein. King [3] developed a oneparameter family of fourth-order methods, which is written as:

$$
\begin{aligned}
& y_{n}=x_{n}-\frac{f\left(x_{n}\right)}{f^{\prime}\left(x_{n}\right)}, \\
& x_{n+1}=y_{n}-\frac{f\left(x_{n}\right)+\beta f\left(y_{n}\right)}{f\left(x_{n}\right)+(\beta-2) f\left(y_{n}\right)} \frac{f\left(y_{n}\right)}{f^{\prime}\left(x_{n}\right)},
\end{aligned}
$$

where $\beta \in \mathbb{R}$ is a constant. In particular, the famous Ostrowski's method [2] is a member of this family for the case $\beta=0$, and it can be written as

$$
\begin{aligned}
& y_{n}=x_{n}-\frac{f\left(x_{n}\right)}{f^{\prime}\left(x_{n}\right)}, \\
& x_{n+1}=y_{n}-\frac{f\left(x_{n}\right)}{f\left(x_{n}\right)-2 f\left(y_{n}\right)} \frac{f\left(y_{n}\right)}{f^{\prime}\left(x_{n}\right)} .
\end{aligned}
$$

Kung and Traub [15] who conjectured that an iteration method without memory based on $n$ evaluations of $f$ or its derivatives could achieve optimal convergence order $2^{n-1}$. Thus, the optimal order for a method with 3 functional evaluations per step would be 4 . King's method [3], Ostrowski's method, and Jarrat's method [16] are some of the optimal fourth-order methods, because they only perform three functional evaluations per step. Recently, based on Ostrowski's or King's methods, some higher-order multipoint methods have been proposed for solving nonlinear equations. Bi et al. developed a scheme of optimal order of convergence eight [17], estimating the first derivative of the function in 
the second and third steps and constructing a weight function as well in the following form:

$$
\begin{aligned}
& y_{n}=x_{n}-\frac{f\left(x_{n}\right)}{f^{\prime}\left(x_{n}\right)}, \\
& z_{n}=y_{n}-\frac{2 f\left(x_{n}\right)-f\left(y_{n}\right)}{2 f\left(x_{n}\right)-5 f\left(y_{n}\right)} \frac{f\left(y_{n}\right)}{f^{\prime}\left(x_{n}\right)}, \\
& x_{n+1}=z_{n}-\frac{f\left(x_{n}\right)+(\gamma+2) f\left(z_{n}\right)}{f\left(x_{n}\right)+\gamma f\left(z_{n}\right)} \\
& \quad \times \frac{f\left(z_{n}\right)}{f\left[z_{n}, y_{n}\right]+f\left[z_{n}, x_{n}, x_{n}\right]\left(z_{n}-y_{n}\right)},
\end{aligned}
$$

where $\gamma \in \mathbb{R}$ is constant. Liu and Wang in [18] presented the following family of optimal order eight:

$$
\begin{aligned}
& y_{n}=x_{n}-\frac{f\left(x_{n}\right)}{f^{\prime}\left(x_{n}\right)}, \\
& z_{n}=y_{n}-\frac{f\left(x_{n}\right)}{f\left(x_{n}\right)-2 f\left(y_{n}\right)} \frac{f\left(y_{n}\right)}{f^{\prime}\left(x_{n}\right)}, \\
& x_{n+1} \\
& =z_{n}-\left[\left(\frac{f\left(x_{n}\right)-f\left(y_{n}\right)}{f\left(x_{n}\right)-2 f\left(y_{n}\right)}\right)^{2}+\frac{f\left(z_{n}\right)}{f\left(y_{n}\right)-\mu f\left(z_{n}\right)}\right. \\
& \left.+\frac{4 f\left(z_{n}\right)}{f\left(x_{n}\right)+\lambda f\left(z_{n}\right)}\right] \frac{f\left(z_{n}\right)}{f^{\prime}\left(x_{n}\right)},
\end{aligned}
$$

where $\mu$ and $\lambda$ are in $\mathbb{R}$. J. R. Sharma and R. Sharma in [12] produce optimal eighth-order method in the following form:

$$
\begin{gathered}
y_{n}=x_{n}-\frac{f\left(x_{n}\right)}{f^{\prime}\left(x_{n}\right)}, \\
z_{n}=y_{n}-\frac{f\left(x_{n}\right)}{f\left(x_{n}\right)-2 f\left(y_{n}\right)} \frac{f\left(y_{n}\right)}{f^{\prime}\left(x_{n}\right)}, \\
x_{n+1}=z_{n}-\left[1+\frac{f\left(z_{n}\right)}{f\left(x_{n}\right)}+\left(\frac{f\left(z_{n}\right)}{f\left(x_{n}\right)}\right)^{2}\right] \\
\times \frac{f\left(z_{n}\right) f\left[x_{n}, y_{n}\right]}{f\left[x_{n}, z_{n}\right] f\left[y_{n}, z_{n}\right]} .
\end{gathered}
$$

In this paper, based on Ostrowski's method, we present a new family of optimal eighth-order methods by using the method of weight functions and we apply a few weight functions to construct families of iterative methods with high convergence and high efficiency index. The convergence analysis is provided to establish their eighth order of convergence. In terms of computational cost, they require the evaluations of only three functions and one first-order derivative per iteration. This gives 1.682 as efficiency index of the presented methods. The new methods are comparable with Bi et al's method, Liu and Wang's method, and Sharma's method. The efficacy of the methods is tested on a number of numerical examples.
We use the symbols $\rightarrow, O$, and $\sim$ according to the following conventions [1]. If $\lim _{x_{n} \rightarrow \infty} g\left(x_{n}\right)=C$, we write $g\left(x_{n}\right) \rightarrow C$ or $g \rightarrow C$. If $\lim _{x \rightarrow a} g(x)=C$, we write $g(x) \rightarrow C$ or $g \rightarrow C$. If $f / g \rightarrow C$, where $C$ is a nonzero constant, we write $f=O(g)$ or $f \sim C g$. Let $f(x)$ be a function defined on an interval $I$, where $I$ is the smallest interval containing $k+1$ distinct nodes $x_{1}, x_{2}, \ldots, x_{k}$. The divided difference $f\left[x_{0}, x_{1}, \ldots, x_{k}\right]$ with the $k$ th order is defined as follows: $f\left[x_{0}\right]=f\left(x_{0}\right)$

$$
\begin{aligned}
f\left[x_{0}\right] & =\frac{f\left[x_{1}\right]-f\left[x_{0}\right]}{x_{1}-x_{0}}, \ldots, f\left[x_{0}, x_{1}, \ldots, x_{k}\right] \\
& =\frac{f\left[x_{1}, x_{2}, \ldots, x_{k}\right]-f\left[x_{0}, x_{1}, \ldots x_{k-1}\right]}{x_{k}-x_{0}} .
\end{aligned}
$$

Moreover, we recall the definition of efficiency index (EI) as $E=p^{1 / n}$, where $p$ is the order of convergence and $n$ is the total number of function evaluations per iteration.

\section{The Methods and Analysis of Convergence}

In order to construct new methods, we consider an iteration scheme of the form

$$
\begin{aligned}
& y_{n}=x_{n}-\frac{f\left(x_{n}\right)}{f^{\prime}\left(x_{n}\right)}, \\
& z_{n}=y_{n}-\frac{f\left(x_{n}\right)}{f\left(x_{n}\right)-2 f\left(y_{n}\right)} \frac{f\left(y_{n}\right)}{f^{\prime}\left(x_{n}\right)}, \\
& x_{n+1}=z_{n}-\frac{f\left(z_{n}\right)}{f^{\prime}\left(z_{n}\right)} .
\end{aligned}
$$

This scheme includes three evaluations of the function and two evaluations of its first derivative. Therefore, this scheme has efficiency index equal to 1.516 . To improve the efficiency index, we approximate $f^{\prime}\left(z_{n}\right)$ by the divided difference [12]

$$
f^{\prime}\left(z_{n}\right) \approx \frac{f\left[x_{n}, z_{n}\right] f\left[y_{n}, z_{n}\right]}{f\left[x_{n}, y_{n}\right]} .
$$

Now, we present a new family of optimal eighth-order Ostrowski-type iterative methods by using the method of weight functions as follows:

$$
\begin{gathered}
y_{n}=x_{n}-\frac{f\left(x_{n}\right)}{f^{\prime}\left(x_{n}\right)}, \\
z_{n}=y_{n}-\frac{f\left(x_{n}\right)}{f\left(x_{n}\right)-2 f\left(y_{n}\right)} \frac{f\left(y_{n}\right)}{f^{\prime}\left(x_{n}\right)}, \\
x_{n+1}=z_{n}-\left\{K\left(t_{1}\right) \times L\left(t_{2}\right) \times P\left(t_{3}\right)\right\} \\
\times \frac{f\left(z_{n}\right) f\left[x_{n}, y_{n}\right]}{f\left[x_{n}, z_{n}\right] f\left[y_{n}, z_{n}\right]},
\end{gathered}
$$

where $K\left(t_{1}\right), L\left(t_{2}\right)$, and $P\left(t_{3}\right)$ are three real-valued weight functions when

$$
t_{1}=\frac{f(z)}{f(x)}, \quad t_{2}=\frac{f(y)}{f(x)}, \quad t_{3}=\frac{f(z)}{f(y)},
$$


without the index $n$, should be chosen such that the order of convergence arrives at the optimal level eight. If $x_{0}$ is an approximation to the zero $\alpha$ of $f$, then the corresponding iterative method is defined by

$$
x_{k+1}=g\left(x_{k}\right), \quad k=0,1, \ldots
$$

Regarding (10), let us define the errors

$$
\begin{gathered}
e=x-\alpha, \quad e_{y}=y-\alpha, \\
e_{z}=z-\alpha, \quad e_{1}=g(x)-\alpha .
\end{gathered}
$$

We will use Taylor's expansion about the zero $\alpha$ to express $f(x), f(y)$, and $f(z)$ as series in $e, e_{y}$, and $e_{z}$, respectively. Then, according to (11), we represent $t_{1}, t_{2}$, and $t_{3}$ as Taylor's polynomials in $e$.

Assume that $x$ is sufficiently close to the zero $\alpha$ of $f$ then $t_{1}, t_{2}$ and $t_{3}$ are close enough to 0 . Let us represent real functions $K, L$, and $P$ appearing in (10) by Taylor's series about 0 ,

$$
\begin{gathered}
K\left(t_{1}\right)=K(0)+K^{\prime}(0) t_{1}+\cdots \\
L\left(t_{2}\right)=L(0)+L^{\prime}(0) t_{2}+\frac{L^{\prime \prime}(0)}{2} t_{2}^{2} \\
+\frac{L^{\prime \prime \prime}(0)}{6} t_{2}^{3}+\frac{L^{(4)}(0)}{24} t_{2}^{4}+\cdots, \\
P\left(t_{3}\right)=P(0)+P^{\prime}(0) t_{3}+\frac{P^{\prime \prime}(0)}{2} t_{3}^{2}+\cdots .
\end{gathered}
$$

Symbolic computations reported here to find candidates for $K, L$, and $P$ have been carried out in a Mathematica 8.0 environment. We will find the coefficients $K(0), K^{\prime}(0), \ldots, P^{\prime \prime}(0)$ of the developments (14) using a simple program in Mathematica 8.0 and an interactive approach explained by the comments C1-C5. First, let us introduce the following abbreviations used in this program (see Algorithm 1):

$$
\begin{aligned}
\mathrm{c}_{\mathrm{k}}=\frac{\mathrm{f}^{(\mathrm{k})}(\alpha)}{\mathrm{k} ! \mathrm{f}^{\prime}(\alpha)}, \quad \mathrm{e}=\mathrm{x}-\alpha, & \mathrm{e}_{1}=\mathrm{g}(\mathrm{x})-\alpha . \\
\mathrm{f}[\mathrm{e}]=\mathrm{f}(\mathrm{x}), & \mathrm{f}\left[\mathrm{e}_{\mathrm{y}}\right]=\mathrm{f}(\mathrm{y}), \quad \mathrm{f}\left[\mathrm{e}_{\mathrm{z}}\right]=\mathrm{f}(\mathrm{z}), \\
\mathrm{f}^{\prime}[\mathrm{e}]=\mathrm{f}^{\prime}(\mathrm{x}), & \mathrm{f} 1 \mathrm{a}=\mathrm{f}^{\prime}(\alpha), \\
\mathrm{K} 0=\mathrm{K}(0), & \mathrm{K} 1=\mathrm{K}^{\prime}(0), \quad \mathrm{K} 2=\mathrm{K}^{\prime \prime}(0), \\
\mathrm{K} 3=\mathrm{K}^{\prime \prime \prime}(0), & \mathrm{K} 4=\mathrm{K}^{(4)}(0), \\
\mathrm{L} 0=\mathrm{L}(0), & \mathrm{L} 1=\mathrm{L}^{\prime}(0), \\
\mathrm{L} 3=\mathrm{L}^{\prime \prime \prime}(0), & \mathrm{L} 4=\mathrm{L}^{(4)}(0), \\
\mathrm{P} 0=\mathrm{P}(0), & \mathrm{P} 1=\mathrm{P}^{\prime \prime}(0), \\
\mathrm{P} 3=\mathrm{P}^{\prime \prime \prime}(0), & \mathrm{P} 4=\mathrm{P}^{(4)}(0) .
\end{aligned}
$$

Comment C1: from the expression of the error $e_{1}=$ $g(x)-\alpha$, we observe that $e_{1}$ is of the form

$$
\begin{aligned}
e_{1} & =g(x)-\alpha \\
& =a_{4} e^{4}+a_{5} e^{5}+a_{6} e^{6}+a_{7} e^{7}+a_{8} e^{8}+O\left(e^{9}\right) .
\end{aligned}
$$

The iterative method $x_{k+1}=g\left(x_{k}\right)$ will have the order of convergence equal to eight if we determine the coefficients of the developments appearing in (14) in such a way that $a_{4}, a_{5}, a_{6}$, and $a_{7}$ (in (16)) vanish. We find these coefficients equalling shaded expressions in boxed formulas to 0. First, from Out [a4], we have

$$
-1+K(0) L(0) P(0)=0 .
$$

Without loss of generality, we can take $K(0)=L(0)=P(0)=$ 1 and hence, $a_{4}=0$. In what follows, $a_{5}, a_{6}, a_{7}$, and $a_{8}$ are calculated using the already found coefficients.

Comment C2: from Out [a5], we see that the choice $L^{\prime}(0)=0$ gives $a_{5}=0$.

0 .

Comment C3: we obtain $a_{6}=0$ choosing $L^{\prime \prime}(0)=P^{\prime}(0)=$

Comment C4: $a_{7}$ vanishes if we choose simultaneously $K^{\prime}(0)=1, L^{\prime \prime \prime}(0)=0$.

Comment C5: substituting the quantities $K(0), K^{\prime}(0), \ldots$, $P^{\prime \prime}(0)$ in the expression of $e_{1}$, found in the described interactive procedure, we obtain

$$
\begin{aligned}
& e_{1}=g(x)-\alpha \\
& =-\frac{1}{24}\left(c_{2}\left(c_{2}^{2}-c_{3}\right)\right. \\
& \quad \times\left(\left(L^{(4)}(0)+12\left(-6+P^{\prime \prime}(0)\right)\right) c_{2}^{4}\right. \\
& \quad-24\left(-4+P^{\prime \prime}(0)\right) c_{2}^{2} c_{3} \\
& \left.\left.\quad+12 P^{\prime \prime}(0) c_{3}^{2}-24 c_{2} c_{4}\right)\right) \\
& \quad \times e_{n}^{8}+O\left(e_{n}^{9}\right)
\end{aligned}
$$

where $\left|L^{\prime \prime \prime}(0)\right|<\infty,\left|P^{\prime \prime}(0)\right|<\infty$.

According to the above analysis, we have proved the following theorem.

Theorem 1. Assume that $f \in C^{5}(D)$. Suppose $\alpha \in D, f(\alpha)=$ 0 and $f^{\prime}(\alpha) \neq 0$. If the initial point $x_{0}$ is sufficiently close to $\alpha$, then the sequence $x_{n}$ generated by any method of the family (10) has eighth order of convergence to $\alpha$ if $K, L$, and $P$ are any functions with

$$
\begin{gathered}
K(0)=K^{\prime}(0)=1, \\
L(0)=1, \quad L^{\prime}(0)=L^{\prime \prime}(0)=L^{\prime \prime \prime}(0)=0, \\
\quad\left|L^{(4)}(0)\right|<\infty, \\
P(0)=1, \quad P^{\prime}(0)=0, \quad\left|P^{\prime \prime}(0)\right|<\infty .
\end{gathered}
$$




$$
\begin{aligned}
& f\left[e_{-}\right]=f 1 a\left(e^{1}+c_{2} e^{2}+c_{3} e^{3}+c_{4} e^{4}+c_{5} e^{5}+c_{6} e^{6}+c_{7} e^{7}+c_{8} e^{8}+c_{9} e^{9}\right) ; \\
& e_{y}=e-\operatorname{Series}\left[\frac{f[e]}{f^{\prime}[e]},\{e, 0,8\}\right] \text {; } \\
& e_{z}=e_{y}-\operatorname{Series}\left[\frac{f[e]}{f[e]-2 f\left[e_{y}\right]} * \frac{f\left[e_{y}\right]}{f^{\prime}[e]},\{e, 0,8\}\right] \text {; } \\
& f\left[x_{-}, y_{-}\right]:=\frac{f[x]-f[y]}{x-y} \text {; } \\
& t_{1}:=\frac{f\left[e_{z}\right]}{f[e]} ; t_{2}:=\frac{f\left[e_{y}\right]}{f[e]} ; t_{3}:=\frac{f\left[e_{z}\right]}{f\left[e_{y}\right]} ; \\
& \mathrm{K}=\mathrm{K} 0+\mathrm{K} 1 * \mathrm{t}_{1}+\frac{1}{2} * \mathrm{~K} 2 * \mathrm{t}_{1}^{2}+\frac{1}{6} * \mathrm{~K} 3 * \mathrm{t}_{1}^{3}+\frac{1}{24} * \mathrm{~K} 4 * \mathrm{t}_{1}^{4} ; \\
& \mathrm{L}=\mathrm{L} 0+\mathrm{L} 1 * \mathrm{t}_{2}+\frac{1}{2} * \mathrm{~L} 2 * \mathrm{t}_{2}^{2}+\frac{1}{6} * \mathrm{~L} 3 * \mathrm{t}_{2}^{3}+\frac{1}{24} * \mathrm{~L} 4 * \mathrm{t}_{2}^{4} \text {; } \\
& \mathrm{P}=\mathrm{P} 0+\mathrm{P} 1 * \mathrm{t}_{3}+\frac{1}{2} * \mathrm{P} 2 * \mathrm{t}_{3}^{2}+\frac{1}{6} * \mathrm{P} 3 * \mathrm{t}_{3}^{3}+\frac{1}{24} * \mathrm{P} 4 * \mathrm{t}_{3}^{4} \\
& e_{1}=e_{z}-\operatorname{Series}\left[K * L * P \frac{f\left[e_{z}\right] f\left[e, e_{y}\right]}{f\left[e, e_{z}\right] f\left[e_{y}, e_{z}\right]},\{e, 0,8\}\right] / / F u l l \text { Simplify } \\
& \text { a4 = Coefficient }\left[e_{1}, e^{4}\right] / / \text { FullSimplify } \\
& \text { C1: Out }[\mathrm{a} 4]=-(-1+\mathrm{KOLOPO}) \mathrm{c}_{2}\left(\mathrm{c}_{2}^{2}-\mathrm{c}_{3}\right) \\
& \mathrm{KO}=1 ; \mathrm{LO}=1 ; \mathrm{PO}=1 ;\left({ }^{*} \text { Vanish coefficient of } e^{4 *}\right) \\
& \text { a5 = Coefficient }\left[e_{1}, e^{5}\right] / / \text { FullSimplify }
\end{aligned}
$$

C2:$$
\text { Out }[\mathrm{a} 5]=(-4+\mathrm{KO}(4 \mathrm{LO}-\mathrm{L} 1) \mathrm{PO}) \mathrm{c}_{2}^{4}+(8+\mathrm{KO}(-8 \mathrm{~L} 0+\mathrm{L} 1) \mathrm{PO}) \mathrm{c}_{2}^{2} \mathrm{c}_{3}
$$$$
+2(-1+\text { KOLOPO }) c_{3}^{2}+2(-1+\text { KOLOPO }) c_{2} c_{4}
$$

L1 $=0$; $\left({ }^{*}\right.$ Vanish coefficient of $\left.e^{5 *}\right)$

a6 = Coefficient $\left[e_{1}, e^{6}\right] / /$ FullSimplify

C3:

$$
\begin{aligned}
\text { Out }[\mathrm{a} 6]=- & \frac{1}{2}(-20+\mathrm{KO}(20 \mathrm{LO}-14 \mathrm{~L} 1+\mathrm{L} 2) \mathrm{PO}+2 \mathrm{KOLOP} 1) \mathrm{c}_{2}^{5} \\
& +\frac{1}{2}(-60+\mathrm{KO}(60 \mathrm{LO}-26 \mathrm{~L} 1+\mathrm{L} 2) \mathrm{PO}+4 \mathrm{KOLOP} 1) \mathrm{c}_{2}^{3} \mathrm{c}_{3} \\
& +(18+\mathrm{KO}(4 \mathrm{~L} 1 \mathrm{PO}-\mathrm{LO}(18 \mathrm{PO}+\mathrm{P} 1))) \mathrm{c}_{2} \mathrm{c}_{3}^{2} \\
& +2(6+\mathrm{KO}(-6 \mathrm{LO}+\mathrm{L} 1) \mathrm{PO}) \mathrm{c}_{2}^{2} \mathrm{c}_{4}+7(-1+\mathrm{KOLOPO}) \mathrm{c}_{3} \mathrm{c}_{4}
\end{aligned}
$$

L2 $=0 ; \mathrm{P} 1=0 ;\left({ }^{*}\right.$ Vanish coefficient of $\left.e^{6 *}\right)$

a7 = Coefficient $\left[e_{1}, e^{7}\right] / /$ FullSimplify

$\begin{aligned} \text { Out }[\mathrm{a} 7]= & -20-\mathrm{K} 1 \mathrm{LOPO}+\mathrm{KO}(21 \mathrm{LOPO}-30 \mathrm{~L} 1 \mathrm{P} 0+5 \mathrm{~L} 2 \mathrm{PO} \\ & \left.\left.-\frac{\mathrm{L} 3 \mathrm{P} 0}{6}+6 \mathrm{LOP} 1-\mathrm{L} 1 \mathrm{P} 1\right)\right) \mathrm{c}_{2}^{6}+\frac{1}{6}(480+12 \mathrm{~K} 1 \mathrm{LOPO} \\ & +\mathrm{K} 0((-492 \mathrm{~L} 0+480 \mathrm{~L} 1-54 \mathrm{~L} 2+\mathrm{L} 3) \mathrm{PO} \\ & +12(-9 \mathrm{~L} 0+\mathrm{L} 1) \mathrm{P} 1)) \mathrm{c}_{2}^{4} \mathrm{c}_{3}+(-80-\mathrm{K} 1 \mathrm{LOPO}+\mathrm{KO}(81 \mathrm{LOPO} \\ & -50 \mathrm{~L} 1 \mathrm{PO}+3 \mathrm{~L} 2 \mathrm{PO}+14 \mathrm{LOP} 1-\mathrm{L} 1 \mathrm{P} 1)) \mathrm{c}_{2}^{2} \mathrm{c}_{3}^{2}+2(6 \\ & +\mathrm{KO}(2 \mathrm{~L} 1 \mathrm{PO}-\mathrm{LO}(6 \mathrm{PO}+\mathrm{P} 1))) \mathrm{c}_{3}^{3}+(-40 \\ & +\mathrm{K} 0(40 \mathrm{LO}-21 \mathrm{~L} 1+\mathrm{L} 2) \mathrm{PO}+4 \mathrm{KOLOP} 1) \mathrm{c}_{2}^{3} \mathrm{c}_{4} \\ & +2(26+\mathrm{KO}(7 \mathrm{~L} 1 \mathrm{PO}-2 \mathrm{LO}(13 \mathrm{PO}+\mathrm{P} 1))) \mathrm{c}_{2} \mathrm{c}_{3} \mathrm{c}_{4} \\ & +6(-1+\mathrm{KOLOPO}) \mathrm{c}_{4}^{2}\end{aligned}$

$\mathrm{K} 1=1 ; \mathrm{L} 3=0 ;\left({ }^{*}\right.$ Vanish coefficient of $\left.e^{7 *}\right)$

$\mathrm{e}_{1} / /$ FullSimplify

C5:

$$
\begin{gathered}
\text { Out }\left[e_{1}\right]=-\frac{1}{24}\left(c _ { 2 } ( c _ { 2 } ^ { 2 } - c _ { 3 } ) \left((L 4+12(-6+P 2)) c_{2}^{4}-24(-4+P 2) c_{2}^{2} c_{3}\right.\right. \\
\left.\left.+12 P 2 c_{3}^{2}-24 c_{2} c_{4}\right)\right) e^{8}+0[e]^{9}
\end{gathered}
$$

$|\mathrm{K} 1|<\infty ;|\mathrm{L} 3|<\infty ;$ 
Remark 2. Any method of the family (10) uses four evaluations per iteration and has eighth-order convergence and satisfies the conditions (19), which accords with the conjecture of Kung-Traub [15] that a multipoint iteration without memory based on $n$ evaluations achieves optimal convergence order $2^{n-1}$ for the case $n=4$.

\section{The Concrete Iterative Methods}

In what follows, we give some concrete iterative forms of scheme (10).

Method 1. The functions $K(t), L(t)$, and $P(t)$ defined by

$$
\begin{gathered}
K(t)=\sin (t)+\cos (t), \\
L(t)=t^{4} e^{t}+1, \quad P(t)=e^{t^{2}}
\end{gathered}
$$

satisfy the conditions of Theorem 1. A new family of twoparameter eighth-order methods is obtained

$$
\begin{gathered}
y_{n}=x_{n}-\frac{f\left(x_{n}\right)}{f^{\prime}\left(x_{n}\right)}, \\
z_{n}=y_{n}-\frac{f\left(x_{n}\right)}{f\left(x_{n}\right)-2 f\left(y_{n}\right)} \frac{f\left(y_{n}\right)}{f^{\prime}\left(x_{n}\right)}, \\
x_{n+1}=z_{n}-\left(\sin \left(\frac{f\left(z_{n}\right)}{f\left(x_{n}\right)}\right)+\cos \left(\frac{f\left(z_{n}\right)}{f\left(x_{n}\right)}\right)\right) \\
\times\left(1+\frac{f^{4}\left(y_{n}\right)}{f^{4}\left(x_{n}\right)} e^{\left(f\left(y_{n}\right) / f\left(x_{n}\right)\right)}\right) e^{\left(f^{2}\left(z_{n}\right) / f^{2}\left(y_{n}\right)\right)} \\
\times \frac{f\left(z_{n}\right) f\left[x_{n}, y_{n}\right]}{f\left[x_{n}, z_{n}\right] f\left[y_{n}, z_{n}\right]},
\end{gathered}
$$

and the error equation becomes

$$
e_{n+1}=c_{2}\left(c_{2}^{2}-c_{3}\right)\left(c_{2}^{4}-2 c_{2}^{2} c_{3}-c_{3}^{2}+c_{2} c_{4}\right) e_{n}^{8}+O\left(e_{n}^{9}\right) \text {. }
$$

Method 2. The functions $K(t), L(t)$, and $P(t)$ defined by

$$
\begin{gathered}
K(t)=e^{t}-1+\cos (t), \\
L(t)=e^{t^{4}}, \quad P(t)=1-t+\sin (t)
\end{gathered}
$$

satisfy the conditions of Theorem 1. A new family of twoparameter eighth-order methods is obtained

$$
\begin{gathered}
y_{n}=x_{n}-\frac{f\left(x_{n}\right)}{f^{\prime}\left(x_{n}\right)}, \\
z_{n}=y_{n}-\frac{f\left(x_{n}\right)}{f\left(x_{n}\right)-2 f\left(y_{n}\right)} \frac{f\left(y_{n}\right)}{f^{\prime}\left(x_{n}\right)},
\end{gathered}
$$

TABLE 1: The examples considered in this study.

\begin{tabular}{lc}
\hline Test functions & Zeros \\
\hline$f_{1}(x)=x^{2}-e^{x}-3 x+2$ & $\alpha \approx 0.25753028543986079$ \\
$f_{2}(x)=x e^{x^{2}}-\sin ^{2}(x)+3 \cos (x)+5$ & $\alpha \approx-1.207647827130919$ \\
$f_{3}(x)=\sin (x) e^{x}+\operatorname{Ln}\left(x^{2}+1\right)$ & $\alpha \approx 0$ \\
$f_{4}(x)=x^{5}+x^{4}+4 x^{2}-15$ & $\alpha \approx 1.3474280989683053$ \\
$f_{5}(x)=10 x e^{-x^{2}}-1$ & $\alpha \approx 1.6796306104284499$ \\
$f_{6}(x)=\cos (x)-x$ & $\alpha \approx 0.73908513321516064$ \\
$f_{7}(x)=e^{-x^{2}+x+2}-1$ & $\alpha \approx-1.000000000000000$ \\
$f_{8}(x)=\operatorname{Ln}\left(x^{2}+x+2\right)-x+1$ & $\alpha \approx 4.1525907367571583$ \\
\hline
\end{tabular}

$$
\begin{aligned}
x_{n+1}= & z_{n}-\left(e^{\left(f\left(z_{n}\right) / f\left(x_{n}\right)\right)}-1+\cos \left(\frac{f\left(z_{n}\right)}{f\left(x_{n}\right)}\right)\right) \\
& \times\left(1-\frac{f\left(z_{n}\right)}{f\left(y_{n}\right)}+\sin \left(\frac{f\left(z_{n}\right)}{f\left(y_{n}\right)}\right)\right) e^{f^{4}\left(y_{n}\right) / f^{4}\left(x_{n}\right)} \\
& \times \frac{f\left(z_{n}\right) f\left[x_{n}, y_{n}\right]}{f\left[x_{n}, z_{n}\right] f\left[y_{n}, z_{n}\right]},
\end{aligned}
$$

and the error equation becomes

$$
e_{n+1}=c_{2}^{2}\left(c_{2}^{2}-c_{3}\right)\left(2 c_{2}^{3}-4 c_{2} c_{3}+c_{4}\right) e_{n}^{8}+O\left(e_{n}^{9}\right) \text {. }
$$

Method 3. The functions $K(t), L(t)$, and $P(t)$ defined by

$$
K(t)=1+\sin (t), \quad L(t)=1+t^{4} \cos (t), \quad P(t)=\cos (t)
$$

satisfy the conditions of Theorem 1. A new family of twoparameter eighth-order methods is obtained

$$
\begin{gathered}
y_{n}=x_{n}-\frac{f\left(x_{n}\right)}{f^{\prime}\left(x_{n}\right)}, \\
z_{n}=y_{n}-\frac{f\left(x_{n}\right)}{f\left(x_{n}\right)-2 f\left(y_{n}\right)} \frac{f\left(y_{n}\right)}{f^{\prime}\left(x_{n}\right)}, \\
x_{n+1}=z_{n}-\left(1+\sin \left(\frac{f\left(z_{n}\right)}{f\left(x_{n}\right)}\right)\right) \\
\times\left(1+\frac{f^{4}\left(y_{n}\right)}{f^{4}\left(x_{n}\right)} \cos \left(\frac{f\left(y_{n}\right)}{f\left(x_{n}\right)}\right)\right) \\
\times \cos \left(\frac{f\left(z_{n}\right)}{f\left(y_{n}\right)}\right) \frac{f\left(z_{n}\right) f\left[x_{n}, y_{n}\right]}{f\left[x_{n}, z_{n}\right] f\left[y_{n}, z_{n}\right]},
\end{gathered}
$$

and the error equation becomes

$$
e_{n+1}=\frac{1}{2} c_{2}\left(c_{2}^{2}-c_{3}\right)\left(5 c_{2}^{4}-10 c_{2}^{2} c_{3}+c_{3}^{2}+2 c_{2} c_{4}\right) e_{n}^{8}+O\left(e_{n}^{9}\right) \text {. }
$$

The families (21), (24), and (27) achieve eighth-order convergence. Per iteration the presented methods require three 
TABLE 2: Comparison of various iterative methods under the same total number of function evaluations $($ TNFE $=12)$.

\begin{tabular}{|c|c|c|c|c|c|c|}
\hline & $(21)$ & (24) & $(27)$ & $(\mathrm{BM})$ & $(\mathrm{LWM})$ & $(\mathrm{ShM})$ \\
\hline \multicolumn{7}{|c|}{$f_{1}, x_{0}=0$} \\
\hline$\left|x_{n}-\alpha\right|$ & $0.23 e-643$ & $0.18 e-654$ & $0.42 e-662$ & $0.46 e-650$ & $0.24 e-613$ & $0.90 e-656$ \\
\hline$\left|f\left(x_{n}\right)\right|$ & $0.85 e-643$ & $0.68 e-654$ & $0.16 e-661$ & $0.17 e-649$ & $0.91 e-613$ & $0.34 e-655$ \\
\hline $\mathrm{COC}$ & 8.000000 & 8.000000 & 8.000000 & 8.000000 & 8.000000 & 8.000000 \\
\hline \multicolumn{7}{|c|}{$f_{2}, x_{0}=-1$} \\
\hline$\left|x_{n}-\alpha\right|$ & $0.24 e-307$ & $0.39 e-293$ & $0.26 e-297$ & $0.48 e-217$ & $0.14 e-340$ & $0.65 e-430$ \\
\hline$\left|f\left(x_{n}\right)\right|$ & $0.49 e-306$ & $0.79 e-292$ & $0.53 e-296$ & $0.97 e-216$ & $0.28 e-339$ & $0.13 e-428$ \\
\hline $\mathrm{COC}$ & 8.000001 & 8.000002 & 8.000002 & 7.999999 & 8.000000 & 8.000000 \\
\hline \multicolumn{7}{|c|}{$f_{3}, x_{0}=2$} \\
\hline$\left|x_{n}-\alpha\right|$ & $0.32 e-83$ & $0.610 e-92$ & $0.74 e-77$ & $0.10 e-107$ & $0.79 e-52$ & $0.14 e-96$ \\
\hline$\left|f\left(x_{n}\right)\right|$ & $0.32 e-83$ & $0.610 e-92$ & $0.74 e-77$ & $0.10 e-107$ & $0.79 e-52$ & $0.14 e-96$ \\
\hline $\mathrm{COC}$ & 7.986895 & 7.993900 & 7.984913 & 7.965999 & 7.899280 & 7.991216 \\
\hline \multicolumn{7}{|c|}{$f_{4}, x_{0}=1.6$} \\
\hline$\left|x_{n}-\alpha\right|$ & $0.12 e-368$ & $0.11 e-359$ & $0.21 e-361$ & $0.66 e-303$ & $0.97 e-307$ & $0.51 e-341$ \\
\hline$\left|f\left(x_{n}\right)\right|$ & $0.45 e-367$ & $0.41 e-358$ & $0.78 e-360$ & $0.24 e-301$ & $0.36 e-305$ & $0.19 e-339$ \\
\hline $\mathrm{COC}$ & 7.999999 & 7.999997 & 8.000018 & 7.999999 & 7.999999 & 8.000000 \\
\hline \multicolumn{7}{|c|}{$f_{5}, x_{0}=1.8$} \\
\hline$\left|x_{n}-\alpha\right|$ & $0.15 e-472$ & $0.13 e-471$ & $0.10 e-485$ & $0.26 e-446$ & $0.47 e-399$ & $0.17 e-444$ \\
\hline$\left|f\left(x_{n}\right)\right|$ & $0.42 e-472$ & $0.36 e-471$ & $0.28 e-485$ & $0.72 e-446$ & $0.13 e-398$ & $0.46 e-444$ \\
\hline $\mathrm{COC}$ & 8.000000 & 8.000000 & 8.000000 & 8.000000 & 8.000000 & 8.000000 \\
\hline \multicolumn{7}{|c|}{$f_{6}, x_{0}=1$} \\
\hline$\left|x_{n}-\alpha\right|$ & $0.11 e-632$ & $0.33 e-572$ & $0.99 e-558$ & $0.98 e-664$ & $0.49 e-581$ & $0.71 e-570$ \\
\hline$\left|f\left(x_{n}\right)\right|$ & $0.18 e-632$ & $0.56 e-572$ & $0.17 e-557$ & $0.16 e-663$ & $0.81 e-581$ & $0.12 e-569$ \\
\hline $\mathrm{COC}$ & 8.000000 & 8.000000 & 8.000000 & 8.000000 & 8.000000 & 8.000000 \\
\hline \multicolumn{7}{|c|}{$f_{7}, x_{0}=-0.5$} \\
\hline$\left|x_{n}-\alpha\right|$ & $0.27 e-235$ & $0.17 e-198$ & $0.84 e-192$ & $0.31 e-221$ & $0.13 e-221$ & $0.35 e-193$ \\
\hline$\left|f\left(x_{n}\right)\right|$ & $0.80 e-235$ & $0.52 e-198$ & $0.25 e-191$ & $0.94 e-221$ & $0.39 e-221$ & $0.11 e-192$ \\
\hline $\mathrm{COC}$ & 8.015338 & 8.000253 & 8.000189 & 8.000058 & 8.000030 & 7.999924 \\
\hline \multicolumn{7}{|c|}{$f_{8}, x_{0}=3.2$} \\
\hline$\left|x_{n}-\alpha\right|$ & $0.31 e-518$ & $0.10 e-485$ & $0.12 e-476$ & $0.19 e-618$ & $0.58 e-487$ & $0.26 e-479$ \\
\hline$\left|f\left(x_{n}\right)\right|$ & $0.19 e-518$ & $0.62 e-486$ & $0.69 e-477$ & $0.11 e-618$ & $0.35 e-487$ & $0.16 e-479$ \\
\hline $\mathrm{COC}$ & 8.000000 & 8.000000 & 8.000000 & 8.000000 & 8.000000 & 8.000000 \\
\hline
\end{tabular}

evaluations of the function and one evaluation of its first derivative. If we assume that all the evaluations have the same cost as function one, we have that the family of methods (10) has the efficiency index of $8^{1 / 4}=1.682$ which is better than $2^{1 / 2}=1.414$ of Newton's method, $4^{1 / 3}=1.587$ of King's methods, $6^{1 / 4}=1.565$ of some methods with sixth-order convergence, and $7^{1 / 4}=1.627$ of some variants of Ostrowski's method with seventh-order convergence [14].

\section{Numerical Results}

Now, Method 1 (21), Method 2 (24), and Method 3 (27) are employed to solve some nonlinear equations and compared with Bi et al's method (BM), (4), (with $\gamma=1$ ), Liu and Wang's method (LWM), (5), (with $\mu=5$ and $\lambda=-7$ ), and Sharma's method (ShM), (6). The test functions of $f(x)$ are listed in Table 1 .
Numerical computations reported here have been carried out in a Mathematica 8.0 environment. Table 2 shows the difference of the root $\alpha$ and the approximation $x_{n}$ to $\alpha$, where $\alpha$ is the exact root computed with 800 significant digits (Digits $:=800$ ) and $x_{n}$ is calculated by using the same total number of function evaluations (TNFE) for all methods. The absolute values of the function $\left(\left|f\left(x_{n}\right)\right|\right)$ and the computational order of convergence (COC) are also shown in Table 2. Here, COC is defined by [19]

$$
\rho \approx \frac{\operatorname{Ln}\left|\left(x_{n+1}-\alpha\right) /\left(x_{n}-\alpha\right)\right|}{\operatorname{Ln}\left|\left(x_{n}-\alpha\right) /\left(x_{n-1}-\alpha\right)\right|} .
$$

\section{Conclusions}

We have obtained a new family of variants of Ostrowski's method. The convergence order of these methods is eight, which consist of three evaluations of the function and one evaluation of the first derivative per iteration, so they have 
an efficiency index equal to $8^{1 / 4}=1.682$. Therefore, the family of methods agrees with the conjecture of Kung-Traub for $n=4$. Numerical examples also show that the numerical results of our new methods, in equal iterations, improve the results of other existing three-step methods with eighth order convergence.

\section{Conflict of Interests}

The authors declare that there is no conflict of interests regarding the publication of this paper.

\section{Acknowledgments}

The authors are grateful to the referees for their comments and suggestions that helped to improve the paper. This research was supported by Islamic Azad University, Hamedan Branch.

\section{References}

[1] J. F. Traub, Iterative Methods for the Solution of Equations, Prentice-Hall, Englewood Cliffs, NJ, USA, 1964.

[2] A. M. Ostrowski, Solution of Equations in Euclidean and Banach Spaces, Academic Press, New York, NY, USA, 3rd edition, 1973.

[3] R. F. King, "A family of fourth order methods for nonlinear equations," SIAM Journal on Numerical Analysis, vol. 10, pp. 876-879, 1973.

[4] F. Soleimani, F. Soleymani, and S. Shateyi, "Some iterative methods free from derivatives and their basins of attraction for nonlinear equations," Discrete Dynamics in Nature and Society, vol. 2013, Article ID 301718, 10 pages, 2013.

[5] G. Fernández-Torres and J. Vásquez-Aquino, “Three new optimal fourth-order iterative methods to solve nonlinear equations," Advances in Numerical Analysis, vol. 2013, Article ID 957496, 8 pages, 2013.

[6] H. Montazeri, F. Soleymani, S. Shateyi, and S. S. Motsa, "On a new method for computing the numerical solution of systems of nonlinear equations," Journal of Applied Mathematics, vol. 2012, Article ID 751975, 15 pages, 2012.

[7] F. Soleymani, "Novel computational iterative methods with optimal order for nonlinear equations," Advances in Numerical Analysis, vol. 2011, Article ID 270903, 10 pages, 2011.

[8] F. Soleymani, S. Karimi Vanani, and A. Afghani, "A general three-step class of optimal iterations for nonlinear equations," Mathematical Problems in Engineering, vol. 2011, Article ID 469512, 10 pages, 2011.

[9] F. Soleymani, M. Sharifi, and B. Somayeh Mousavi, "An improvement of Ostrowski's and King's techniques with optimal convergence order eight," Journal of Optimization Theory and Applications, vol. 153, no. 1, pp. 225-236, 2012.

[10] F. Soleymani, S. Karimi Vanani, and M. Jamali Paghaleh, "A class of three-step derivative-free root solvers with optimal convergence order," Journal of Applied Mathematics, vol. 2012, Article ID 568740, 15 pages, 2012.

[11] F. Soleymani, S. K. Vanani, M. Khan, and M. Sharifi, "Some modifications of King's family with optimal eighth order of convergence," Mathematical and Computer Modelling, vol. 55, no. 3-4, pp. 1373-1380, 2012.
[12] J. R. Sharma and R. Sharma, "A new family of modified Ostrowski's methods with accelerated eighth order convergence," Numerical Algorithms, vol. 54, no. 4, pp. 445-458, 2010.

[13] C. Chun and Y. Ham, "Some sixth-order variants of Ostrowski root-finding methods," Applied Mathematics and Computation, vol. 193, no. 2, pp. 389-394, 2007.

[14] J. Kou, Y. Li, and X. Wang, "Some variants of Ostrowski's method with seventh-order convergence," Journal of Computational and Applied Mathematics, vol. 209, no. 2, pp. 153-159, 2007.

[15] H. T. Kung and J. F. Traub, "Optimal order of one-point and multipoint iteratione," Journal of the ACM, vol. 21, no. 4, pp. 643-651, 1974.

[16] P. Jarrat, "Some fourth order multipoint iterative methods for solving equations," Mathematical Computation, vol. 20, pp. 434437, 1966.

[17] W. Bi, Q. Wu, and H. Ren, "A new family of eighth-order iterative methods for solving nonlinear equations," Applied Mathematics and Computation, vol. 214, no. 1, pp. 236-245, 2009.

[18] L. Liu and X. Wang, "Eighth-order methods with high efficiency index for solving nonlinear equations," Applied Mathematics and Computation, vol. 215, no. 9, pp. 3449-3454, 2010.

[19] S. Weerakoon and T. G. I. Fernando, "A variant of Newton's method with accelerated third-order convergence," Applied Mathematics Letters, vol. 13, no. 8, pp. 87-93, 2000. 


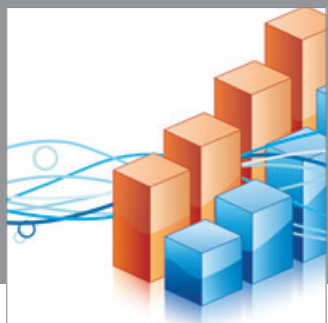

Advances in

Operations Research

mansans

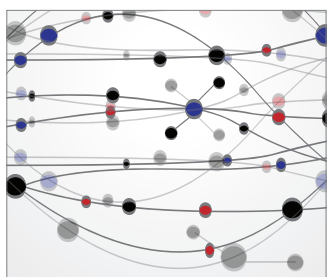

The Scientific World Journal
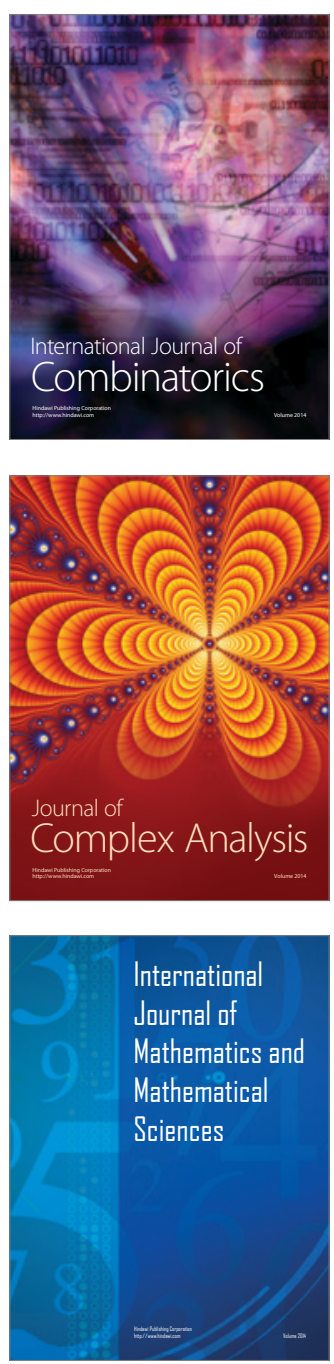
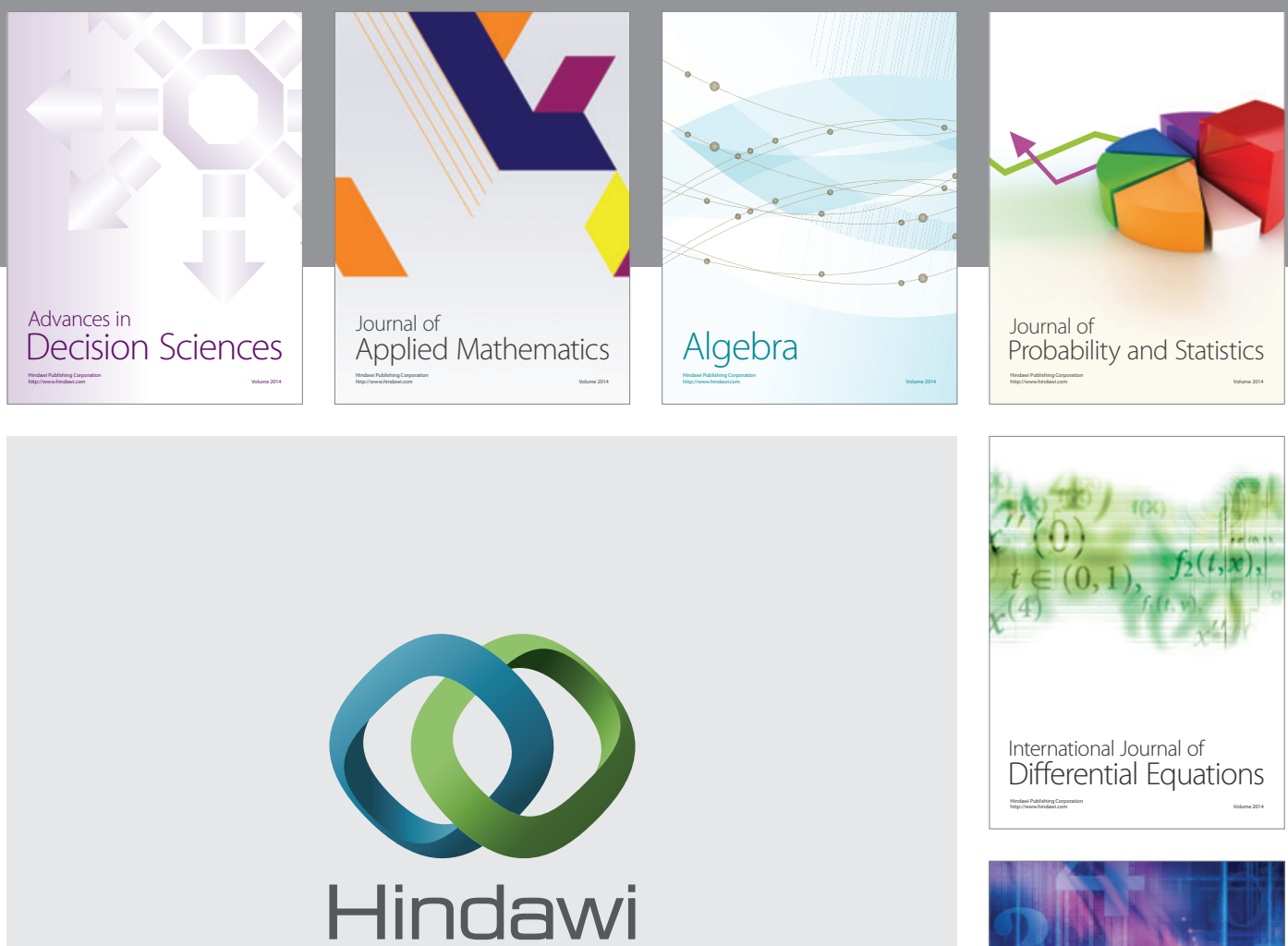

Submit your manuscripts at http://www.hindawi.com
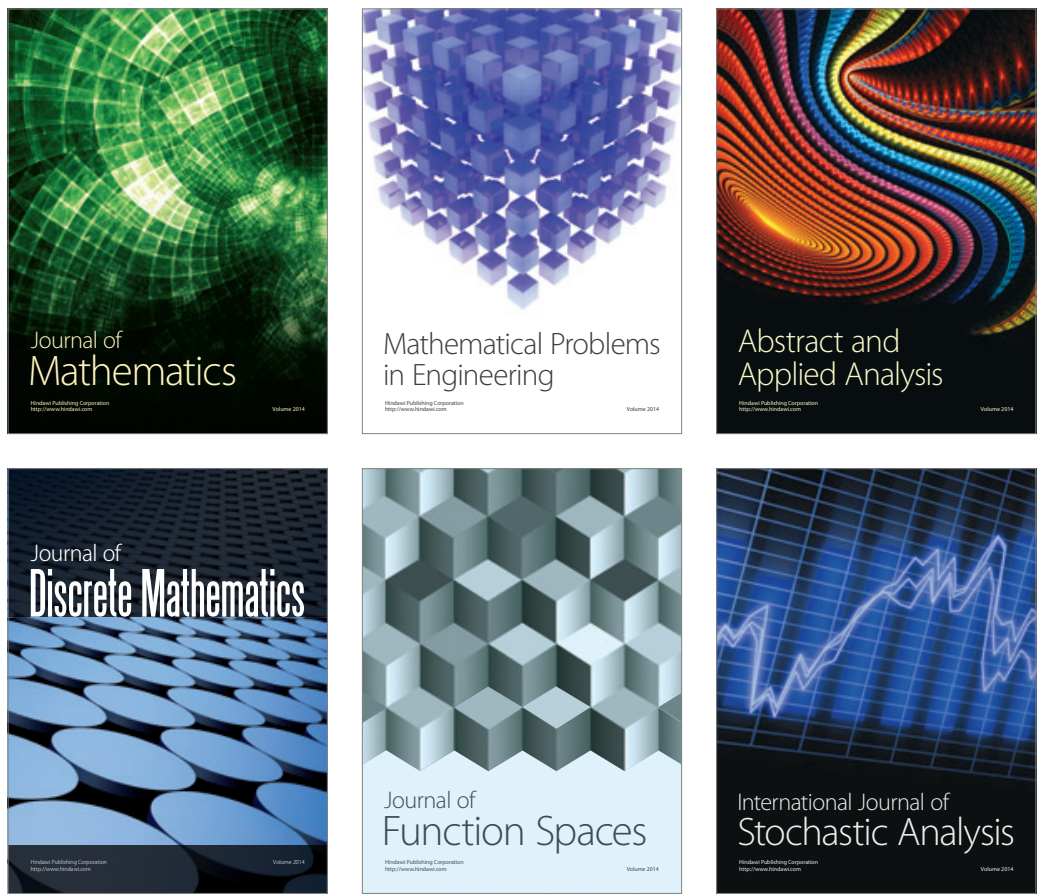

Journal of

Function Spaces

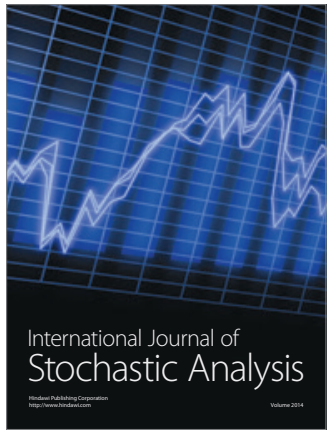

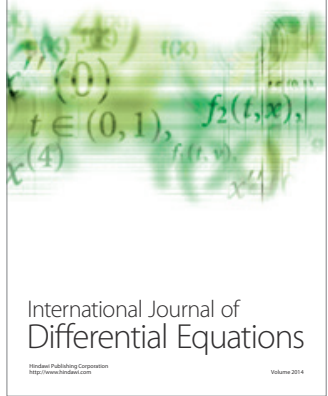
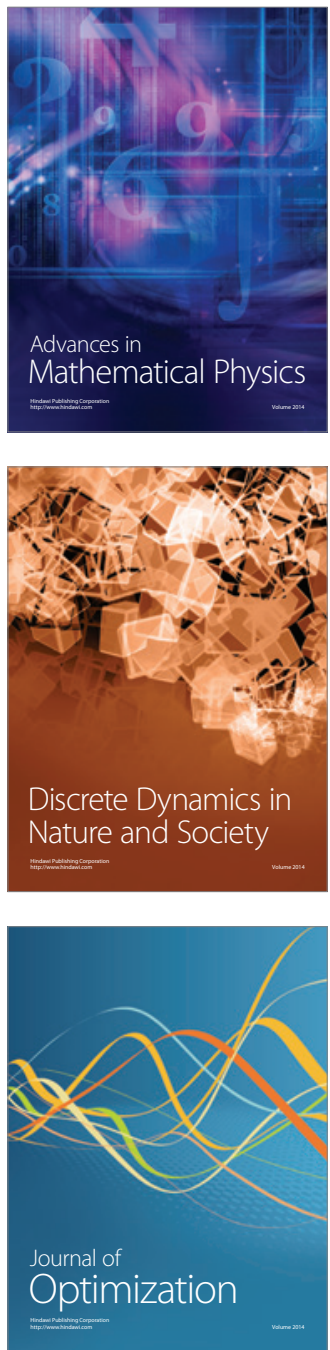